\title{
Recurrence-associated factors of laparoscopic adenomyomectomy for severely symptomatic adenomyoma
}

\author{
WENTAO YU ${ }^{1,2}$, GUANYUAN LIU ${ }^{1}$, CHONGDONG LIU ${ }^{1}$ and ZHENYU ZHANG ${ }^{1}$ \\ ${ }^{1}$ Department of Obstetrics and Gynecology, Beijing Chao-Yang Hospital, Capital Medical University, \\ Beijing 10020, P.R. China; ${ }^{2}$ Department of Vascular Surgery, Brigham and Women's Hospital, \\ Harvard Medical School, Boston, MA 02115, USA
}

Received December 10, 2017; Accepted May 18, 2018

DOI: $10.3892 / \mathrm{ol} .2018 .9082$

\begin{abstract}
The present study aimed to identify which patients with adenomyoma would benefit from sparing the uterus and which patients should undergo a hysterectomy to avoid secondary surgery. Patients with pathology-proven adenomyoma admitted to Beijing Chao-Yang Hospital between November 2005 and November 2015 were retrospectively reviewed. Relief and reappearance of dysmenorrhea following laparoscopic adenomyomectomy were evaluated. All 49 patients (mean age, 40.6 \pm 5.2 years; age range, 26-51 years) presented with severe dysmenorrhea prior to surgery. Dysmenorrhea was identified to be relieved in $83.7 \%$ (41/49) of patients at the 6-month follow-up. No factors were revealed to have a significant effect on the surgical outcome. The median follow-up period was 4.6 (1-11) years; and 24.5\% (12/49) of patients experienced recurrence of dysmenorrhea. Multivariate analysis identified preoperative serum cancer antigen 125 (CA 125) levels [hazard ratio (HR), 2.356; 95\% confidence interval (CI), 1.271-3.570; $\mathrm{P}=0.011]$, postoperative gonadotropin-releasing hormone agonist (GnRH-a) treatment (HR, 0.540; 95\% CI, 0.241-0.873; $\mathrm{P}=0.017$ ) and accompanying endometriosis (HR, 2.182; 95\% CI, 1.556-3.031; $\mathrm{P}=0.003$ ) as independent risk factors for relapse. Laparoscopic adenomyomectomy is effective for alleviating dysmenorrhea in patients with adenomyoma. Patients with lower preoperative serum CA 125 levels without accompanying endometriosis benefited greater from adenomyomectomy compared with all
\end{abstract}

Correspondence to: Dr Zhenyu Zhang, Department of Obstetrics and Gynecology, Beijing Chao-Yang Hospital, Capital Medical University, 8 Gongti South Street, Beijing 10020, P.R. China

E-mail: zhenyuzhang2000@163.com

Abbreviations: CA 125, cancer antigen 125; GnRH-a, gonadotropin-releasing hormone agonist; LNG-IUS, levonorgestrel-releasing intrauterine system; LPSN, laparoscopic presacral neurectomy; ROC, receiver-operating characteristic; UNA, uterine nerve ablation; VAS, visual analogue scale

Key words: adenomyosis, dysmenorrhea, laparoscopy, pelvic pain, uterus other patients. Postoperative GnRH-a treatment strengthens therapeutic effects.

\section{Introduction}

Uterine adenomyosis is a benign gynecological condition characterized by the ectopic growth of the endometrial tissue, including the endometrial glands and stroma, located at least $2.5 \mathrm{~mm}$ below the endometrial-myometrial junction (1). The two main forms of adenomyosis are diffuse and focal adenomyoma. Diffuse adenomyosis is more common and characterized by the foci of endometrial mucosa scattered throughout the uterine musculature. Adenomyoma is uncommon, but not rare, and is defined as encapsulated foci including the two glands and stroma implanted within the myometrium (2). Adenomyoma is mostly solid and rarely manifests as cystic $(2,3)$. In the majority of cases, the border between the lesion and the surrounding myometrium is not clear. Therefore, the term 'adenomyoma' may be interpreted as grossly circumscribed adenomyotic masses (4). Medical therapy for adenomyosis may be effective. Unfortunately, the effects are often transient, and symptoms and signs usually reappear when therapy is stopped (5). For decades, hysterectomy was the most popular therapeutic option for patients with symptomatic adenomyosis $(6,7)$. Hysterectomy may be a curative treatment, but it is not acceptable to all women (8). Numerous women want alternatives to traditional hysterectomy. However, there are no evidence-based guidelines regarding the appropriate treatment of symptomatic uterine adenomyosis in patients who want to preserve the uterus (5). Furthermore, few reports have focused on factors affecting recurrence following laparoscopic adenomyomectomy. The present study aimed to help physicians identify patients with adenomyoma who would benefit from uterus sparing and those who should undergo hysterectomy to avoid a secondary surgery.

\section{Materials and methods}

Patients. Ethical approval for the collection and publication of patient data was obtained from the institutional review board of Beijing Chao-Yang Hospital of Capital Medical University (Beijing, China; reference no. EC1335097) and written informed consent was obtained from all participating 
patients. The medical records of 49 female patients (mean age, 40.6 \pm 5.2 years; age range, 26-51 years) diagnosed with adenomyoma confirmed by postoperative pathology at Beijing Chao-Yang Hospital of Capital Medical University, admitted between November 2005 and November 2015, were retrospectively reviewed. All inclusion and inclusion criteria are presented in Fig. 1. The following baseline information was collected: Age, body weight, body height, obstetric history, gynecologic history, smoking history, drinking history, pathological uterine volume (uterus volume was determined by the following formula: $\mathrm{a} \times \mathrm{b} \times \mathrm{c} \times \pi / 6$, where $\mathrm{a}, \mathrm{b}$ and $\mathrm{c}$ indicate maximum length, width and thickness of the uterus, respectively), serum cancer antigen 125 (CA 125) level, visual analogue scale (VAS) (9) score on admission, number of adenomyomas, and other uterine or ovarian pathologies accompanying adenomyosis. All patients received 3-course injections of gonadotropin-releasing hormone agonist (GnRH-a) prior to surgery to reduce the uterine volume and bleeding during surgery. If the patients reported menorrhagia, diagnostic curettage was performed to exclude any malignancy 2 weeks prior to surgery. All patients received transvaginal ultrasound evaluations prior to surgery. An experienced ultrasound doctor was available, however, due to the relatively high cost of the magnetic resonance imaging (MRI), only 14 patients underwent MRI prior to surgery. Representative ultrasound and MRI images of adenomyomas are presented in Figs. 2 and 3.

Patients were asked to specify a VAS score for dysmenorrhea 6 months subsequent to the completion of all therapies. The patients were divided into 3 groups based on this score: Mild or no pain (VAS score 0-2), moderate pain (VAS score 3-6) and severe pain (VAS score 7-10). All patients were followed up at 6 and 12 months subsequent to completing all therapies during a clinical visit. Thereafter, they were followed up at yearly intervals starting from the second year through a phone conversation or outpatient clinic visit, all the patients were followed up till menopause or June 2017 in this article. The endpoint is when the patients entered their menopause. The mean follow-up period was 4.6 years. Reevaluations of the VAS rating and transvaginal ultrasound examination were performed during the outpatient clinic visit if they experienced reappearance or aggravation of dysmenorrhea. As the time when dysmenorrhea relapsed after surgery varied among patients, it was difficult to define a cutoff time for the reappearance of dysmenorrhea the shortest remission period was 7 months, while in some patients it was several years or there was no relapse of dysmenorrhea at all. Recurrence was determined by the subjective VAS score of the patients throughout the follow-up duration following a period of remission from surgery once all other potential causes were excluded. A total of 53 patients fulfilled the inclusion criteria and 4 patients were excluded due to loss to follow-up; therefore, 49 patients were eligible for analysis. Approximately $60 \%$ of the leiomyomas and adenomyomas were located at the posterior wall of uterus, $30 \%$ were located at the anterior wall and $10 \%$ were at the fundus uteri. Tumor position was not included in the present study as it was revealed that relapses did not always appear at the original site where the adenomyosis was removed. Occasionally, relapse occurred on the opposite side of the uterus to where the surgery was performed. It was difficult to identify from where the relapses originated from, particularly for patients with $>1$ lesion prior to surgery.

The operative technique involved the recognition of the position of the lesion by careful inspection of the uterus. This is usually difficult and requires adequate experience as the borders of an adenomyoma are not well-defined compared with that of a fibroid (1). Then, an incision was created on the uterine wall with monopolar diathermy or tissue scissors. Next, gradual dissection of the adenomyoma was performed with scissors, monopolar diathermy and/or bipolar diathermy without penetrating the endometrium. All recognizable lesions were removed. Surrounding healthy myometrium was removed if necessary. Then, closure of the uterine serosa and myometrium was performed. Finally, intraoperative frozen biopsy was performed to exclude the uterine sarcoma. A total of 8 patients underwent LPSN (Laparoscopic presacral neurectomy) during laparoscopic adenomyomectomy. After identifying the sacral promontory, the peritoneum overlying the sacral promontory was elevated and incised vertically, the retroperitoneal fatty tissue was then removed, the hypogastric plexus was elevated to the lateral side, the nerve fibers are coagulated and excised from the sacral promontory level. The removed nerve tissue was sent for histologic examination.

Statistical analysis. Data are presented as the mean \pm standard deviation. Statistical analyses were conducted using SPSS statistical package for Windows (version 21.0; IBM Corp., Armonk, NY, USA). An unpaired Student's t-test and one-way analysis of variance with Fisher's Least Significant Difference post hoc test were used to compare continuous variables. Categorical variables were examined using a $\chi^{2}$ test and a Kruskal-Wallis test. The optimum cut-off points for CA 125 were determined by the receiver-operating characteristic (ROC) curves for recurrence prediction. Univariate relapse analyses results were evaluated using the Kaplan-Meier method and compared using the log-rank test. Multivariate analyses involving Cox regression models were performed to determine independent factors associated with recurrence. $\mathrm{P}<0.05$ was considered to indicate a statistically significant difference.

\section{Results}

Prior to surgery, all patients reported severe dysmenorrhea with a VAS score of $9.12 \pm 1.05$, whereas 16 women had simultaneous menorrhagia. Seven patients were nulliparous, and each of the others experienced only 1 successful birth (except for 1 patient who gave birth twice). The patients had no previous history of gynecological surgery except 13 patients who experienced cesarean deliveries. The preoperative serum CA 125 level was $80.50 \pm 56.78 \mathrm{U} / \mathrm{ml}$. Endometriosis was confirmed in 23 patients, and 14 had uterine leiomyoma confirmed by postoperative pathology examination. Eight (16.3\%) subjects underwent laparoscopic presacral neurectomy (LPSN) and laparoscopic adenomyomectomy. Twenty-six (53.1\%) patients were prescribed 6 months of postoperative GnRH-a therapy. Eight (16.3\%) patients were prescribed a levonorgestrel-releasing intrauterine system (LNG-IUS). For patients who selected GnRH-a or LNG-IUS therapy following surgery, the first injection of postoperative GnRH-a was 


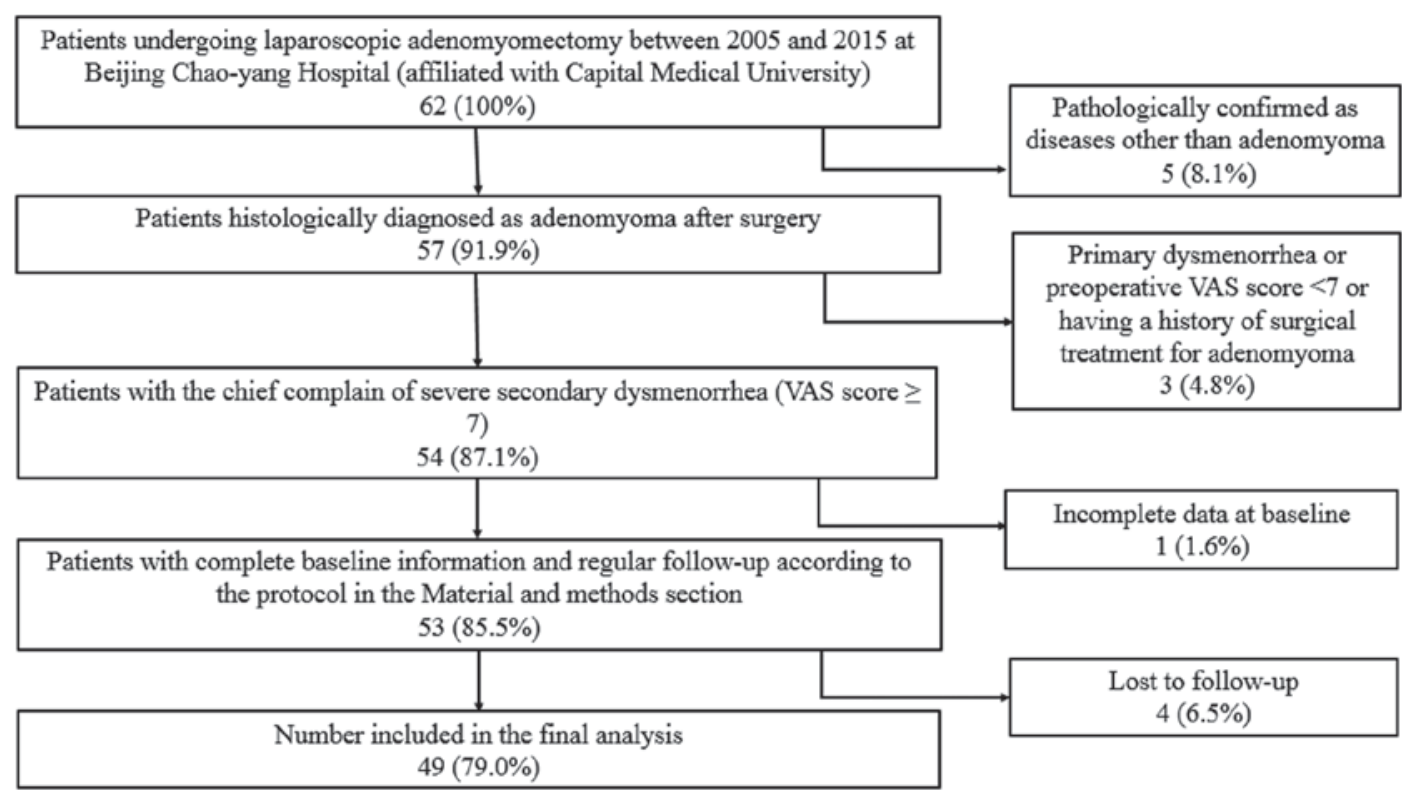

Figure 1. Patient enrollment flowchart. VAS, visual analogue scale.

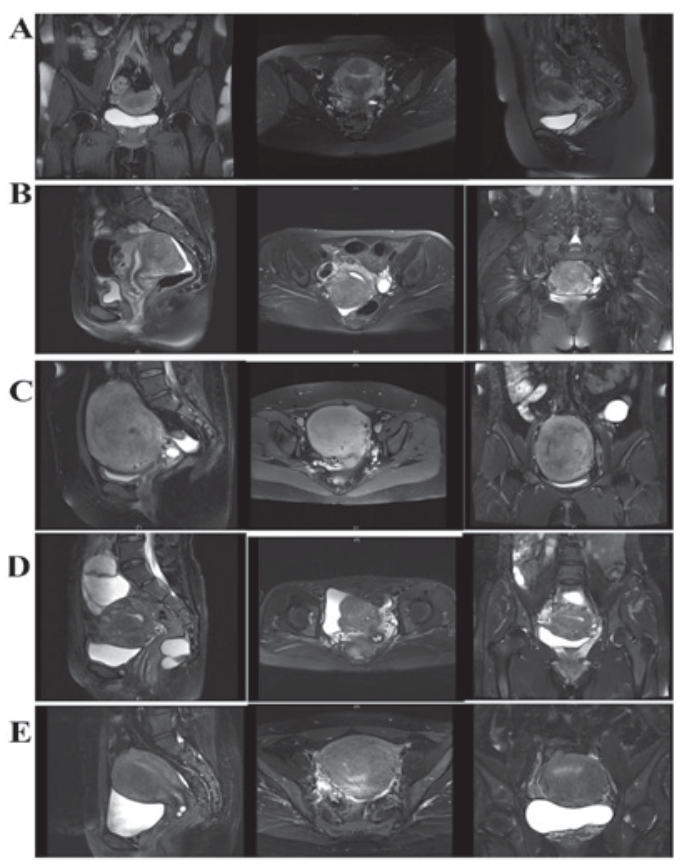

Figure 2. Representative magnetic resonance image of 5 different patients with adenomyoma prior to surgery. Adenomyomas were located at (A) posterior wall and fundus (B) Posterior wall, (C) fundus, (D) anterior wall and fundus and (E) anterior wall.

administered immediately following surgery. LNG-IUS was administered at 3-6 months following surgery, depending on the excision extent and depth of the uterine wall, to avoid perforation. Adjuvant treatments were administered according to the patients' personal preferences. There were no major complications following surgery. Postoperative pain alleviation was evaluated by the patient's self-assessment based on abatement of dysmenorrhea at the 6-month follow-up visit. Forty-one patients experienced varying degrees of reduced symptoms of dysmenorrhea, with an overall effectiveness rate of $83.7 \%$ (41/49). Twenty-eight (57.1\%) patients had mild or

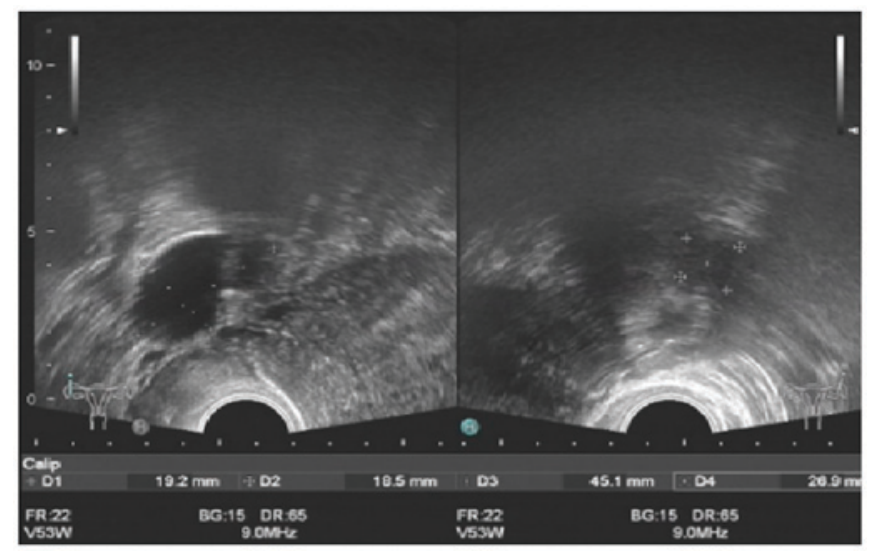

Figure 3. Ultrasound images of one patient with adenomyoma during relapse.

no pain subsequent to treatment and had VAS scores ranging between 0 and 2. Menorrhagia improved in $68.8 \%(11 / 16)$ of patients. Associations between potential factors and the mitigation of dysmenorrhea were evaluated by univariate analysis. The p-VAS was significantly different among different groups while no factor was found significantly related to mitigation of dysmenorrhea following resection (Table I).

Dysmenorrhea ratings during the follow-up period are summarized in Table II. Of the 49 patients, $24.5 \%$ (12/49) experienced relapse at the end of the follow-up period. The mean diagnosis of recurrence occurred 18.5 (6-34) months after resection. Of the 12 patients who relapsed, seven patients were in the mild or no pain group and 5 were in the moderate pain group at 6 months of follow-up. A total of $4(33.3 \%)$ experienced relapse within the first year following treatment, 3 (25\%) experienced relapse during the second year and $5(41.7 \%)$ experienced relapse during the third year subsequent to treatment. The VAS score of one patient following the reappearance of dysmenorrhea reached 8 , which was higher compared with the preoperative assessment score. The patient underwent uterine 
Table I. Factors influencing dysmenorrhea relief as determined by p-VAS.

\begin{tabular}{|c|c|c|c|c|}
\hline Variables & $\begin{array}{l}\text { No relief } \\
\quad(n=8)\end{array}$ & $\begin{array}{c}\text { Partial } \\
\text { relief }(n=13)\end{array}$ & $\begin{array}{l}\text { Near-complete } \\
\text { relief }(n=28)\end{array}$ & P-value \\
\hline $\mathrm{p}$-VAS & $9.38 \pm 0.74$ & $4.46 \pm 0.97$ & $0.46 \pm 0.69$ & $<0.001$ \\
\hline Age (years) & $41.4 \pm 6.8$ & $40.6 \pm 4.7$ & $40.3 \pm 5.1$ & 0.884 \\
\hline Body Mass Index $\left(\mathrm{kg} / \mathrm{m}^{2}\right)$ & $22.3 \pm 3.5$ & $22.8 \pm 2.5$ & $23.1 \pm 3.3$ & 0.831 \\
\hline Menorrhagia & & & & 0.369 \\
\hline Yes, \% (n) & $12.5 \%(2)$ & $43.8 \%(7)$ & $43.8 \%(7)$ & \\
\hline No, \% (n) & $18.2 \%(6)$ & $18.2 \%(6)$ & $63.6 \%(21)$ & \\
\hline Gravidity & $1.8 \pm 1.0$ & $1.8 \pm 1.0$ & $2.5 \pm 1.0$ & 0.055 \\
\hline History of cesarean delivery & & & & 0.558 \\
\hline Yes, \% (n) & $23.1 \%(3)$ & $7.7 \%(1)$ & $69.2 \%(9)$ & \\
\hline No, \% (n) & $13.9 \%(5)$ & $33.3 \%(12)$ & $52.8 \%(19)$ & \\
\hline Preoperative serum cancer antigen 125 level (U/ml) & $84.9 \pm 79.2$ & $91.6 \pm 47.9$ & $74.1 \pm 54.6$ & 0.647 \\
\hline Pathological uterine size $\left(\mathrm{cm}^{3}\right)$ & $127.0 \pm 58.2$ & $145.9 \pm 68.3$ & $145.5 \pm 93.1$ & 0.845 \\
\hline No of adenomyomas & & & & 0.919 \\
\hline Single, $\%(\mathrm{n})$ & $18.2 \%(4)$ & $22.7 \%(5)$ & $59.1 \%(13)$ & \\
\hline Multiple, \% (n) & $14.8 \%(4)$ & $29.6 \%(8)$ & $55.6 \%(15)$ & \\
\hline Coexisting endometriosis & & & & 0.203 \\
\hline Yes, \% (n) & $8.7 \%(2)$ & $26.1 \%(6)$ & $65.2 \%(15)$ & \\
\hline No, $\%(n)$ & $23.1 \%(6)$ & $26.9 \%(7)$ & $50.0 \%(13)$ & \\
\hline Accompanying uterine leiomyoma & & & & 0.344 \\
\hline Yes, $\%(\mathrm{n})$ & $14.2 \%(2)$ & $42.9 \%(6)$ & $42.9 \%(6)$ & \\
\hline No, $\%(n)$ & $17.1 \%(6)$ & $20.0 \%(7)$ & $62.9 \%(22)$ & \\
\hline Laparoscopic presacral neurectomy & & & & 0.796 \\
\hline Yes, \% (n) & $12.5 \%(1)$ & $37.5 \%(3)$ & $50.0 \%(4)$ & \\
\hline No, \% (n) & $17.1 \%(7)$ & $24.4 \%(10)$ & $58.5 \%(24)$ & \\
\hline Postoperative gonadotropin-releasing hormone agonist therapy & & & & 0.566 \\
\hline Yes, $\%(\mathrm{n})$ & $19.2 \%(5)$ & $26.9 \%(7)$ & $53.9 \%(14)$ & \\
\hline No, \% (n) & $13.0 \%(3)$ & $26.1 \%(6)$ & $60.9 \%(14)$ & \\
\hline Postoperative levonorgestrel-releasing intrauterine system treatment & & & & 0.503 \\
\hline Yes, \% (n) & $25 \%(2)$ & $0 \%(0)$ & $75 \%(6)$ & \\
\hline No, \% (n) & $14.6 \%(6)$ & $31.7 \%(13)$ & $53.7 \%(22)$ & \\
\hline
\end{tabular}

Data are presented as mean \pm standard deviation. $\mathrm{p}$-VAS, postoperative visual analogue scale.

artery embolization at Peking Union Medical College Hospital (Beijing, China). The VAS score of another patient increased from 4 to 7 at 17 months following resection; therefore, she underwent a hysterectomy. The degree of pain for the remaining 10 patients experiencing relapse did not reach or exceeded the preoperative level, and they chose oral analgesics when required. Of the 8 patients whose symptoms were not relieved following surgery, 5 were referred for additional surgery (4 hysterectomies and 1 laparoscopic adenomyomectomy). Potential risk factors influencing postoperative recurrence were evaluated by univariate analysis and reported in Table III. Age at surgery $(\mathrm{P}=0.017)$, preoperative serum $\mathrm{CA} 125$ level $(\mathrm{P}=0.012)$ and postoperative $\mathrm{GnRH}$-a therapy $(\mathrm{P}=0.025)$ were identified as significant risk factors for relapse of dysmenorrhea. Patients in the recurrence group tended to be younger, had higher preoperative serum CA 125 level and fewer of them chose to receive GnRH-a therapy following surgery compared with the recurrence free group.

The optimum cut-off point of the preoperative serum CA 125 level for the prediction of relapse was identified as $46.2 \mathrm{U} / \mathrm{ml}$, with a maximum joint sensitivity of $100 \%$ and specificity of 54\% using ROC curve analysis (Fig. 4). Based on this cut-off point, all recurrences were observed for patients with preoperative serum CA 125 levels $>46.2 \mathrm{U} / \mathrm{ml}$, and all recurrent cases had a baseline serum CA 125 level $>46.2$ U/ml (Fig. 5A). Furthermore, 53.1\% (26/49) of subjects received 6 courses of postoperative $\mathrm{GnRH}$-a injections as additional therapy; of these, $11.5 \%$ (3/26) experienced relapse compared with $39.1 \%(9 / 23)$ of those who did not undergo GnRH-a treatment. Kaplan-Meier curves revealed significant differences $(\mathrm{P}=0.025)$ regarding recurrence between $\mathrm{GnRH}-\mathrm{a}$ injection group and non-GnRH-a injection group (Fig. 5B). Univariate Kaplan-Meier curves revealed no significant 
Table II. Dysmenorrhea rating during the follow up period.

\begin{tabular}{|c|c|c|c|c|c|}
\hline \multirow[b]{2}{*}{$\begin{array}{l}\text { Follow-up } \\
\text { period (months) }\end{array}$} & \multirow[b]{2}{*}{$\begin{array}{c}\text { No of } \\
\text { patients }\end{array}$} & \multirow[b]{2}{*}{$\begin{array}{l}\text { Visual analogue } \\
\text { scale rating }\end{array}$} & \multicolumn{3}{|c|}{ Groups (no. of patients) } \\
\hline & & & $\begin{array}{c}0-2 \\
\text { (mild or no pain) }\end{array}$ & $\begin{array}{c}3-6 \\
\text { (moderate pain) }\end{array}$ & $\begin{array}{c}\text { 7-10 } \\
\text { (severe pain) }\end{array}$ \\
\hline Preoperative & 49 & $9.12 \pm 1.05$ & 0 & 0 & 49 \\
\hline 6 & 49 & $2.98 \pm 3.42$ & 28 & 13 & 8 \\
\hline 12 & 49 & $3.16 \pm 3.27$ & 25 & 15 & 9 \\
\hline 24 & 44 & $3.32 \pm 3.38$ & 21 & 15 & 8 \\
\hline 36 & 35 & $4.11 \pm 3.54$ & 13 & 13 & 9 \\
\hline 48 & 30 & $3.97 \pm 3.41$ & 11 & 13 & 6 \\
\hline 60 & 22 & $3.77 \pm 3.18$ & 8 & 11 & 3 \\
\hline 72 & 12 & $3.33 \pm 2.64$ & 4 & 7 & 1 \\
\hline 84 & 8 & $4.38 \pm 2.13$ & 1 & 6 & 1 \\
\hline 96 & 5 & $5.20 \pm 1.48$ & 0 & 4 & 1 \\
\hline 108 & 2 & $4.00 \pm 1.41$ & 0 & 2 & 0 \\
\hline 120 & 1 & 5 & 0 & 1 & 0 \\
\hline
\end{tabular}

Data are presented as the mean \pm standard deviation.

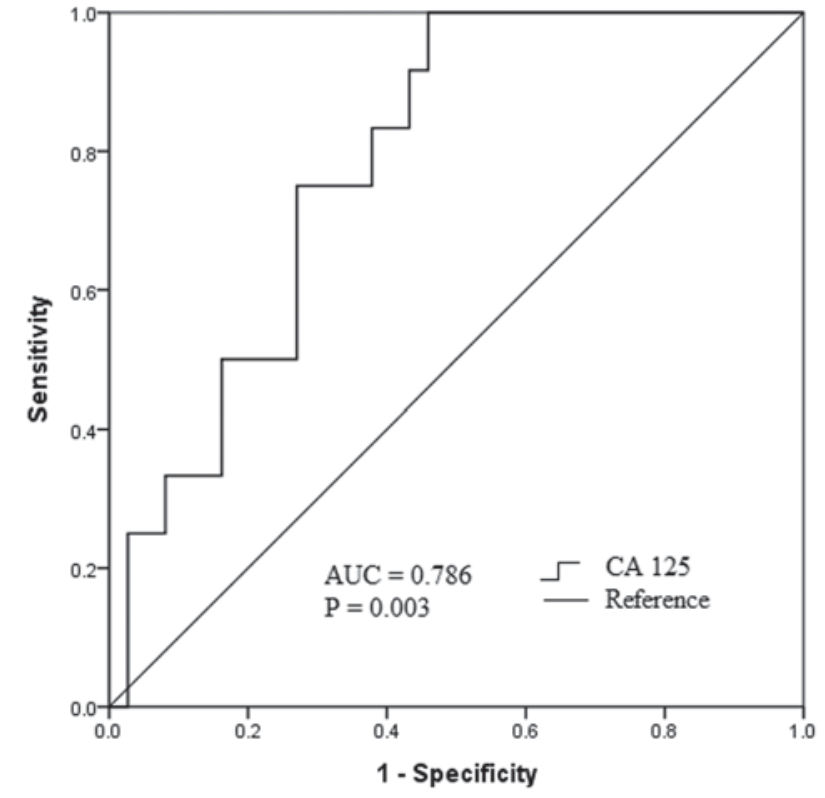

Figure 4. ROC curve analysis of the optimum predictive value of CA 125 for relapse. The AUC was 0.786 (95\% confidence interval, 0.658-0.914), with a maximum joint sensitivity of $100 \%$ and specificity of $54 \%$ at $46.2 \mathrm{U} / \mathrm{ml}$. ROC, receiver-operating characteristic; CA 125, cancer antigen 125; AUC, area under curve.

association between accompanying endometriosis or myoma and relapse (Fig. 5C and D). A Cox proportional hazard model indicated that the preoperative serum CA 125 level [hazard ratio (HR), 2.356; 95\% confidence interval (CI), 1.271-3.570; $\mathrm{P}=0.011$, postoperative $\mathrm{GnRH}$-a treatment (HR, 0.540; 95\% CI, 0.241-0.873; $\mathrm{P}=0.017)$ and accompanying endometriosis (HR, 2.182; 95\% CI, 1.556-3.031; $\mathrm{P}=0.003$ ) were significant risk factors associated with dysmenorrhea relapse following laparoscopic adenomyomectomy (Fig. 6).
Of the 7 nulliparous women, 3 had no desire to have children and 2 underwent in vitro fertilization that failed. Two patients $(28.6 \%)$ became pregnant and experienced successful childbirth during the 2-year follow-up period. Other parous women did not attempt to become pregnant.

\section{Discussion}

Adenomyomectomy includes steps similar to those of myomectomy, except that the margin between the tumor and surrounding normal myometrium may not be as substantial as those of myomectomy (10). This procedure is suitable for patients presenting with focal-type adenomyosis, which may be relatively clearly separated from adjacent normal tissue. This type of surgery was performed during the present study. Other researchers have attempted modifications including U-shaped suturing, overlapping muscle flap suturing and the triple-flap method (11). Cytoreductive surgery involves the excision of diffuse adenomyosis and is similar to the treatment of advanced ovarian cancer types. This procedure is primarily useful when it is difficult to remove all adenomyotic foci (1). The boundary between the adenomyoma and myometrium was ill-defined. A recent review indicated that recurrence rates of adenomyosis differed depending on the extent of excision (12). Different modifications include the transverse $\mathrm{H}$ incision technique, wedge resection of the uterus and asymmetric uterine dissection (13-15).

There are few reports on the factors that influence the recurrence of adenomyosis. In the present study, it was revealed that patients with adenomyosis with higher CA 125 levels and accompanying endometriosis were more likely to experience the relapse of dysmenorrhea following adenomyomectomy. Preoperative serum CA 125 appeared to be a promising predictor of relapse, with a cut-off value of $46.2 \mathrm{U} / \mathrm{ml}$ in the present study. CA 125 is a glycoprotein 
Table III. Univariate analysis of variables influencing recurrence in enrolled patients.

\begin{tabular}{|c|c|c|c|}
\hline Characteristics & No recurrence $(n=37)$ & Recurrence $(\mathrm{n}=12)$ & P-value \\
\hline Age (years) & $41.6 \pm 5.2$ & $37.5 \pm 4.1$ & $0.017^{\mathrm{a}}$ \\
\hline Body mass index $\left(\mathrm{kg} / \mathrm{m}^{2}\right)$ & $23.1 \pm 2.7$ & $22.1 \pm 4.1$ & 0.302 \\
\hline Menorrhagia & & & 0.315 \\
\hline Yes, \% (n) & $25 \%(2)$ & $53.8 \%(7)$ & \\
\hline No, $\%(\mathrm{n})$ & $75 \%(6)$ & $46.2 \%(6)$ & \\
\hline Gravidity & $2.1 \pm 1.0$ & $2.4 \pm 1.1$ & 0.393 \\
\hline History of cesarean delivery & & & 0.999 \\
\hline Yes, \% (n) & $37.5 \%(3)$ & $7.7 \%(1)$ & \\
\hline No, $\%(n)$ & $62.5 \%(5)$ & $92.3 \%(12)$ & \\
\hline Preoperative serum cancer antigen 125 level (U/ml) & $69.0 \pm 54.3$ & $115.8 \pm 51.0$ & $0.012^{\mathrm{a}}$ \\
\hline Pathological uterine size $\left(\mathrm{cm}^{3}\right)$ & $135.4 \pm 84.0$ & $164.5 \pm 70.1$ & 0.285 \\
\hline No of adenomyomas & & & 0.683 \\
\hline Single, $\%(\mathrm{n})$ & $72.7 \%(16)$ & $27.3 \%(6)$ & \\
\hline Multiple, \% (n) & $77.8 \%(21)$ & $22.2 \%(6)$ & \\
\hline Coexisting endometriosis & & & 0.363 \\
\hline Yes, \% (n) & $69.6 \%(16)$ & $30.4 \%(7)$ & \\
\hline No, $\%(n)$ & $80.8 \%(21)$ & $19.2 \%(5)$ & \\
\hline Accompanying uterine leiomyoma & & & 0.156 \\
\hline Yes, \% (n) & $92.9 \%(13)$ & $7.1 \%(1)$ & \\
\hline No, \% (n) & $68.6 \%(24)$ & $31.4 \%(11)$ & \\
\hline Laparoscopic presacral neurectomy & & & 0.999 \\
\hline Yes, \% (n) & $75 \%(6)$ & $25 \%(2)$ & \\
\hline No, \% (n) & $75.6 \%(31)$ & $24.4 \%(10)$ & \\
\hline Postoperative gonadotropin-releasing hormone agonist therapy & & & $0.025^{\mathrm{a}}$ \\
\hline Yes, \% (n) & $88.5 \%(23)$ & $11.5 \%(3)$ & \\
\hline No, \% (n) & $60.9 \%(14)$ & $39.1 \%(9)$ & \\
\hline Postoperative levonorgestrel-releasing intrauterine system treatment & & & 0.190 \\
\hline Yes, $\%(\mathrm{n})$ & $100 \%(8)$ & $0 \%(0)$ & \\
\hline No, \% (n) & $78.4 \%(29)$ & $21.6 \%(12)$ & \\
\hline
\end{tabular}

Data are presented as the mean \pm standard deviation. ${ }^{\mathrm{a}} \mathrm{P}<0.05$.

derived from the embryonic coelomic epithelium and is expressed by certain normal tissue types. High-serum CA 125 has been commonly used as a biomarker for epithelial ovarian cancer $(16,17)$. Increased serum CA 125 serves as a diagnostic tool for screening adenomyosis; however, it lacks specificity in its ability to differentiate adenomyosis from other diseases (18). Kil et al (16) measured serum CA 125 in 2,149 women diagnosed with adenomyosis who underwent total hysterectomy and revealed that it was promising in the differential diagnosis of adenomyosis and leiomyoma at $19 \mathrm{U} / \mathrm{ml}$, with a sensitivity of $66 \%$ and specificity of $79.3 \%$. Using $19 \mathrm{U} / \mathrm{ml}$ as a reference, there may be a proportion of patients suitable for adenomyomectomy screened with a serum CA level of $46.2 \mathrm{U} / \mathrm{ml}$ in clinical practice. Although the mechanism of increased CA 125 is not yet fully understood, inflammatory reactions, peritoneal irritation or peritoneal stretch that alter endothelial permeability may allow CA 125 to reach the circulation, thereby resulting in higher CA 125 concentrations (17). In addition, the ectopic endometrium was confirmed to secrete significantly higher CA 125 concentrations compared with the normal endometrium in patients with adenomyosis (18). Furthermore, serum levels of CA 125 were higher in moderate to severe cases of endometriosis compared with in mild cases (19). A previous study revealed that suppressing CA 125 may suppress the adhesion, invasion and migration of ovarian cancer cells (20), thereby implying that CA 125 may facilitate ectopic endometrium migration and adhesion in the surrounding myometrium during adenomyosis development.

Postoperative GnRH-a was identified as an independent factor influencing relapse in the present study. GnRH-a therapy results in the decreased secretion of gonadotropin, which results in the suppression of the hypothalamicpituitary-gonadal axis and ovarian function, and additionally results in hypoestrogenism, which inhibits the proliferative action of the myometrium (5). Entopic and ectopic types of 

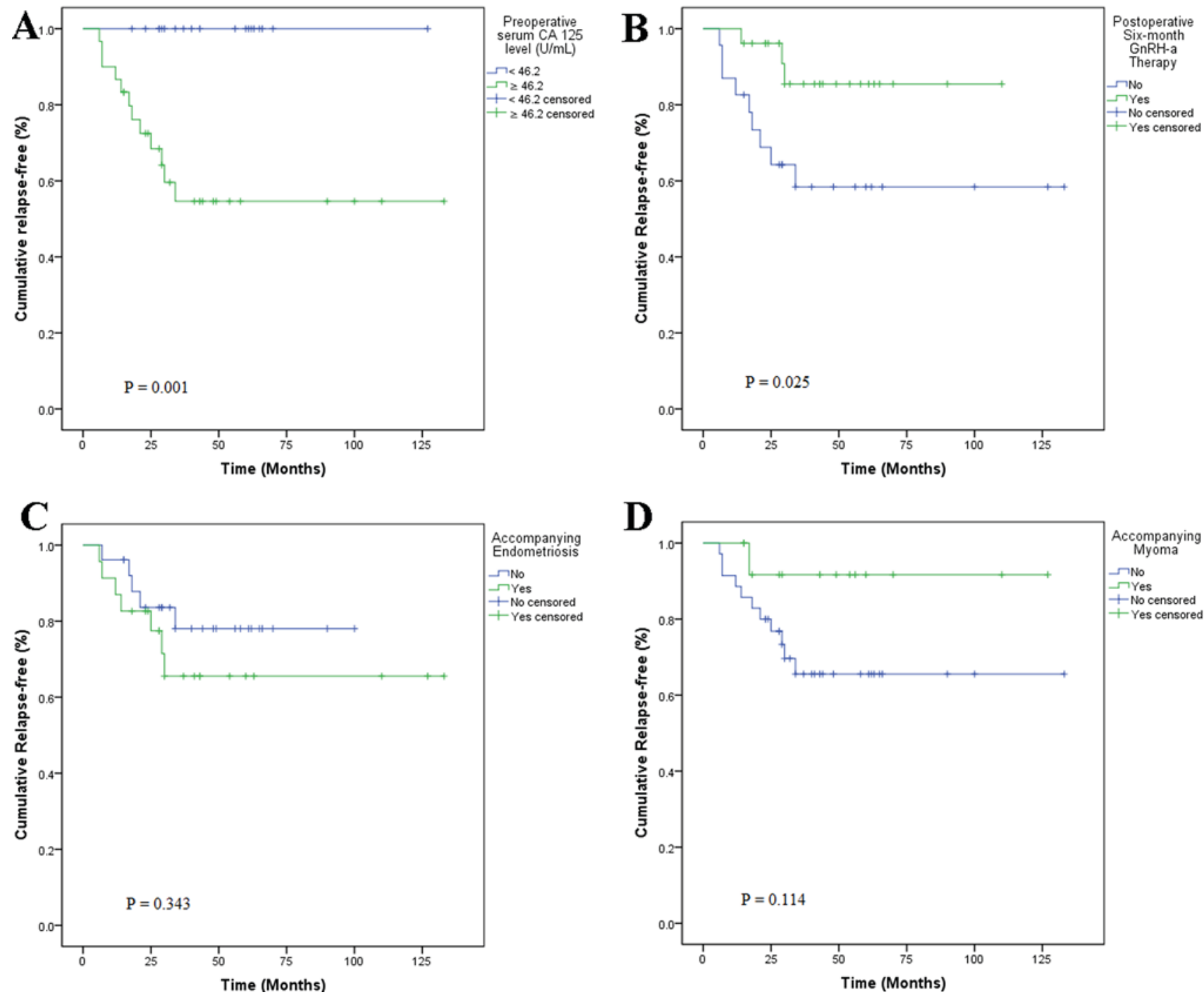

Figure 5. Kaplan-Meier curves for time to relapse subsequent to adenomyomectomy. (A) Patients with preoperative serum CA 125 levels $\geq 46.2 \mathrm{U} / \mathrm{ml}$ and $<46.2 \mathrm{U} / \mathrm{ml}$. (B) Patients who did or did not receive GnRH-a therapy postoperatively for 6 months. Patients with accompanying (C) endometriosis or (D) myoma. CA 125, cancer antigen 125; GnRH-a, gonadotropin-releasing hormone agonist.

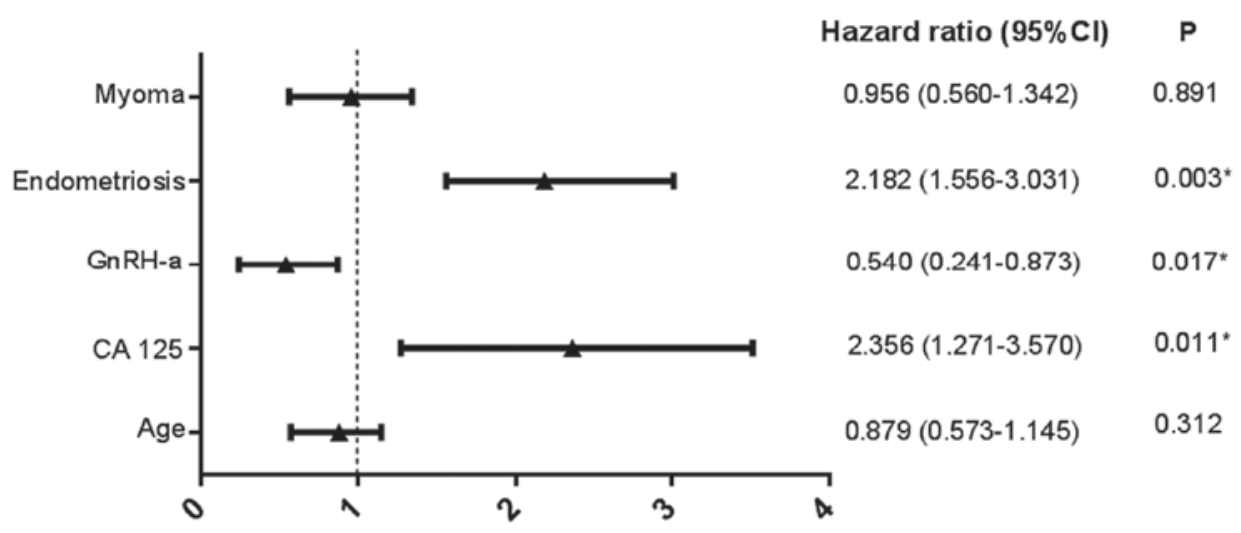

Figure 6. Multivariable Cox regression analysis of risk factors associated with relapse. ${ }^{*} \mathrm{P}<0.05$. CI, confidence interval; CA 125, cancer antigen 125; GnRH-a, gonadotropin-releasing hormone agonist.

endometria of adenomyosis exhibit a series of molecular and metabolic changes that increase angiogenesis and proliferation, enhance local progesterone resistance and augment estrogen production (7). Thereby, increasing infiltration and excessive growth of endometrial stromal cells in the junctional zone facilitates the establishment of adenomyosis (21). GnRH-a therapy has been demonstrated to reduce angiogenesis and inflammation and to induce apoptosis in women 
diagnosed with adenomyosis (21). In the present study, 26 of 49 women received 6 courses of GnRH-a therapy; and of this group, $11.5 \%(3 / 26)$ experienced relapse at the end of the follow-up period whereas $39.1 \%(9 / 23)$ experienced relapse in the surgical-only treatment group. Instead of surgery alone, 6 courses of postoperative administration of GnRH-a should be suggested to prevent relapse following adenomyomectomy. It was considered that the development of adenomyosis may be the consequence of interactions between promotive and inhibitive factors. Promotive factors including higher serum CA 125 concentrations, higher estrogen production and resistance to progesterone may facilitate adenomyotic pathological processes including the migration of ectopic endometrium and proliferation of endometrial stromal cells in the junctional zone. Inhibitive factors including $\mathrm{GnRH}-\mathrm{a}$ therapy and decreased estrogen production prevent this process (7). Therefore, it was hypothesized that GnRH-a may curb this process and delay time to recurrence. Relapse may be a matter of time due to exposure to a pathological environment prior to menopause (22).

Patients with adenomyosis with accompanying endometriosis compared with patients with accompanying myoma were revealed to be more likely experience relapse in the present study. Endometriosis shares a range of common features with adenomyosis, from clinical manifestations to etiology (7). It was hypothesized that they were different forms of the same pathogenesis; however, previous evidence revealed specific distinct pathogenic pathways for adenomyosis, suggesting that they were two different entities (23). Compared with adenomyosis, endometriosis appeared to be more frequent in young nulliparous women, but adenomyosis occurred in relatively older and parous women. One previous report states that adenomyosis and deep endometriosis often coexist, particularly in women with infertility or dysmenorrhea (24); however, opposing views were held that adenomyosis and deep endometriosis do not necessarily coexist $(25,26)$ and the exact incidence of endometriosis in adenomyosis remains unknown. Eight patients received LPSN during adenomyomectomy in the present study. The relapse rates in the two groups that received or did not receive this procedure were similar, and no significant associations between performing LPSN and relapse of pain were identified. One strategy for treating dysmenorrhea and pelvic pain was to prevent the conduction of pain to the nerve center. Uterine nerve ablation (UNA) and PSN were the two most commonly used procedures. UNA involves cutting the nerves along the uterosacral ligament. Although pelvic endometriosis often coexists with adenomyosis, the efficacy of UNA may decrease over time due to limited denervation (27). PSN involves cutting the presacral nerve from the sacral promontory level; therefore, cutting more nerves compared with UNA may be more effective for pain control. Eight patients in the present study underwent PSN during the first several years of the present study. Although PSN and UNA are effective for alleviating pain, technical difficulties and complications arise from affected visceral functions including constipation or urinary urgency. Therefore, these procedures were cautiously applied as adjuvant therapy in the present study.

The present study had a number of limitations. There was the potential for patient selection bias due to the nature of retrospective analysis. Patients with incomplete data were excluded. Not all potentially confounding factors that may have affected the outcomes were obtained from the onset. As a tertiary referral institution, Beijing Chao-Yang Hospital of Capital Medical University admitted patients with relatively complex conditions more easily, whereas other less complicated patients may were referred to other non-tertiary hospitals due to a referral bias. Due to the degree of dysmenorrhea was based on the patient's subjective judgment, and therefore differences in the experiences of patients may have affected the results. In addition, a relatively small number of samples and heterogeneity among subjects may have limited the power of the statistical tests, resulting in false conclusions.

In conclusion, adenomyomectomy may be the treatment of choice in patients with adenomyoma who have lower preoperative serum CA 125 levels and without accompanying endometriosis.

\section{Acknowledgements}

Not applicable.

\section{Funding}

The present study was supported by the International Science \& Technology Cooperation Program of China (grant no. 2012DFR30490).

\section{Availability of data and materials}

The datasets used and/or analyzed during the current study are available from the corresponding author on reasonable request.

\section{Authors' contributions}

WY and CL conceived and designed the study. WY, GL, CL and $\mathrm{ZZ}$ acquired, analyzed and interpreted the raw data. $\mathrm{ZZ}$ revised the manuscript critically for important intellectual content. $\mathrm{ZZ}$ provided final approval of the version to be published.

\section{Ethics approval and consent to participate}

Approval for collection and publication of patient data was obtained from the institutional review board of Beijing Chao-Yang Hospital of Capital Medical University (Beijing, China; reference no. EC1335097), and written informed consent was obtained from all participating patients.

\section{Patient consent for publication}

The patient, or parent, guardian or next of kin provided written informed consent for the publication of any associated data and accompanying images.

\section{Competing interests}

The authors declare that they have no competing interests. 


\section{References}

1. Struble J, Reid S and Bedaiwy MA: Adenomyosis: A clinical review of a challenging gynecologic condition. J Minim Invasive Gynecol 23: 164-185, 2016.

2. Brosens I, Gordts S, Habiba M and Benagiano G: Uterine cystic adenomyosis: A disease of younger women. J Pediatr Adolesc Gynecol 28: 420-426, 2015.

3. Takeuchi H, Kitade M, Kikuchi I, Kumakiri J, Kuroda K and Jinushi M: Diagnosis, laparoscopic management, and histopathologic findings of juvenile cystic adenomyoma: A review of nine cases. Fertil Steril 94: 862-868, 2010.

4. Tahlan A, Nanda A and Mohan H: Uterine adenomyoma: A clinicopathologic review of 26 cases and a review of the literature. Int J Gynecol Pathol 25: 361-365, 2006.

5. Pontis A, D'Alterio MN, Pirarba S, de Angelis C, Tinelli R and Angioni S: Adenomyosis: A systematic review of medical treatment. Gynecol Endocrinol 32: 696-700, 2016.

6. Yavuzcan A, Başbuğ A, Baştan M, Çağlar M and Özdemir İ: The effect of adenomyosis on the outcomes of laparoscopic hysterectomy. J Turk Ger Gynecol Assoc 17: 150-154, 2016.

7. Garcia L and Isaacson K: Adenomyosis: Review of the literature. J Minim Invasive Gynecol 18: 428-437, 2011.

8. Alabiso G, Alio L, Arena S, di Prun AB, Bergamini V, Berlanda N, Busacca M, Candiani M, Centini G, Cello AD, et al: Adenomyosis: What the patient needs. J Minim Invasive Gynecol 23: 476-488, 2016.

9. Langley GB and Sheppeard H: The visual analogue scale: Its use in pain measurement. Rheumatol Int 5: 145-148, 1985.

10. Wang PH, Liu WM, Fuh JL, Cheng MH and Chao HT: Comparison of surgery alone and combined surgical-medical treatment in the management of symptomatic uterine adenomyoma. Fertil Steril 92: 876-885, 2009.

11. Grimbizis GF, Mikos T and Tarlatzis B: Uterus-sparing operative treatment for adenomyosis. Fertil Steril 101: 472-487, 2014.

12. Younes G and Tulandi T: Conservative surgery for adenomyosis and results: A systematic review. J Minim Invasive Gynecol 25 265-276, 2018

13. Fujishita A, Masuzaki H, Khan KN, Kitajima M and Ishimaru T: Modified reduction surgery for adenomyosis. A preliminary report of the transverse $\mathrm{H}$ incision technique. Gynecol Obstet Invest 57: 132-138, 2004.

14. Sun AJ, Luo M, Wang W, Chen R and Lang JH: Characteristics and efficacy of modified adenomyomectomy in the treatment of uterine adenomyoma. Chin Med J (Engl) 124: 1322-1326, 2011.

15. Nishida M, Takano K, Arai Y, Ozone H and Ichikawa R: Conservative surgical management for diffuse uterine adenomyosis. Fertil Steril 94: 715-719, 2010.

16. Kil K, Chung JE, Pak HJ, Jeung IC, Kim JH, Jo HH and Kim MR: Usefulness of CA125 in the differential diagnosis of uterine adenomyosis and myoma. Eur J Obstet Gynecol Reprod Biol 185: 131-135, 2015.
17. Seeber B, Sammel MD, Fan X, Gerton GL, Shaunik A, Chittams J and Barnhart KT: Panel of markers can accurately predict endometriosis in a subset of patients. Fertil Steril 89: 1073-1081, 2008.

18. Kobayashi H, Ida W, Terao T and Kawashima Y: Molecular characteristics of the CA 125 antigen produced by human endometrial epithelial cells: Comparison between eutopic and heterotopic epithelial cells. Am J Obstet Gynecol 169: 725-730, 1993.

19. Mihalyi A, Gevaert O, Kyama CM, Simsa P, Pochet N, De Smet F, De Moor B, Meuleman C, Billen J, Blanckaert N, et al: Non-invasive diagnosis of endometriosis based on a combined analysis of six plasma biomarkers. Hum Reprod 25: 654-664, 2010.

20. Bast RC Jr, Hennessy B and Mills GB: The biology of ovarian cancer: New opportunities for translation. Nat Rev Cancer 9: 415-428, 2009

21. Khan KN, Kitajima M, Hiraki K, Fujishita A, Sekine I, Ishimaru T and Masuzaki H: Changes in tissue inflammation, angiogenesis and apoptosis in endometriosis, adenomyosis and uterine myoma after GnRH agonist therapy. Hum Reprod 25: 642-653, 2010.

22. Jee BC, Lee JY, Suh CS, Kim SH, Choi YM and Moon SY: Impact of GnRH agonist treatment on recurrence of ovarian endometriomas after conservative laparoscopic surgery. Fertil Steril 91: 40-45, 2009.

23. Vannuccini S, Tosti C, Carmona F, Huang SJ, Chapron C, Guo SW and Petraglia F: Pathogenesis of adenomyosis: An update on molecular mechanisms. Reprod Biomed Online 35: 592-601, 2017.

24. Gonzalez-Ramos R, Rocco J, Rojas C, Sovino H, Poch A, Kohen P, Alvarado-Díaz C and Devoto L: Physiologic activation of nuclear factor kappa-B in the endometrium during the menstrual cycle is altered in endometriosis patients. Fertil Steril 97: 645-651, 2012 .

25. Naftalin J, Hoo W, Pateman K, Mavrelos D, Holland T and Jurkovic D: How common is adenomyosis? A prospective study of prevalence using transvaginal ultrasound in a gynaecology clinic. Hum Reprod 27: 3432-3439, 2012.

26. Benagiano G, Brosens I and Habiba M: Structural and molecular features of the endomyometrium in endometriosis and adenomyosis. Hum Reprod Update 20: 386-402, 2014.

27. Johnson NP,FarquharCM,Crossley S, Yu Y, Van Peperstraten AM, Sprecher M and Suckling J: A double-blind randomised controlled trial of laparoscopic uterine nerve ablation for women with chronic pelvic pain. BJOG 111: 950-959, 2004.

This work is licensed under a Creative Commons Attribution-NonCommercial-NoDerivatives 4.0 International (CC BY-NC-ND 4.0) License. 